Louisiana State University

LSU Digital Commons

$1-1-2017$

\title{
Observations of GW170817 by DESGW and the DECam GW-EM Collaboration
}

James Annis

Fermi National Accelerator Laboratory

G. González

R. Hynes

Follow this and additional works at: https://digitalcommons.Isu.edu/physics_astronomy_pubs

\section{Recommended Citation}

Annis, J., González, G., \& Hynes, R. (2017). Observations of GW170817 by DESGW and the DECam GW-EM Collaboration. Proceedings of the International Astronomical Union, 13 (S338), 72-79. https://doi.org/ $10.1017 /$ S1743921318000935

This Conference Proceeding is brought to you for free and open access by the Department of Physics \& Astronomy at LSU Digital Commons. It has been accepted for inclusion in Faculty Publications by an authorized administrator of LSU Digital Commons. For more information, please contact ir@lsu.edu. 


\title{
Observations of GW170817 by DESGW and the DECam GW-EM Collaboration
}

\author{
James Annis ${ }^{1}$ \\ ${ }^{1}$ Fermi National Accelerator Laboratory \\ Batavia, Illinois, USA \\ email: annis@fnal.gov
}

\begin{abstract}
On August 17, 2017 LIGO/Virgo detected a binary neutron star via gravitational waves. We observed 70 sq-degrees in the LIGO/Virgo spatial localization with the DECam on the $4 \mathrm{~m}$ Blanco telescope covering $80 \%$ of the final map. Our group independently discovered an optical counterpart in NGC 4993. We searched our entire imaged region: the object in NGC 4993 was the only viable candidate. Our observations of NGC4993 show complicated morphology but simple star formation history. Our x-ray and radio observations indicate an off-axis jet as afterglow. Our high-cadence optical and infrared spectra show a source that must be described by at least two components, one of which is dominated by the r-process nucleosynthesis elements characteristic of a kilonova. Our modeling of the light curve demonstrates such a model in which $0.05 \mathrm{M}_{\odot}$ of material is ejected from the system. Finally, we discuss the first standard siren measurement of $\mathrm{H}_{0}$.
\end{abstract}

Keywords. gravitational waves, (cosmology:) cosmological parameters,

\section{Introduction}

The DESGW and the DECam GW-EM Collaboration use of the Dark Energy Camera (DECam; Flaugher et al. 2015), a $3.1 \mathrm{deg}^{2}$ wide-field imager on the prime focus of the Blanco $4 \mathrm{~m}$ telescope at the Cerro Tololo Inter-American Observatory, to perform wide area searches for the counterparts of LIGO/Virgo gravitational wave (GW) sources.

DESGW performs the search and discovery. LIGO/Virgo has published two triggered binary black hole (BBH) mergers from their observing run O1: GW150914 and GW151226, and three from O2: GW170104, GW170608, and GW170814 (respectively Abbott et al. 2016, Abbott et al. 2017a, Abbott et al. 2017c, Abbott et al. 2017d, and Abbott et al. 2017e). We have published DECam based optical searches on GW150914 (Soares-Santos et al. 2016, Annis et al. 2016), GW151226 (Cowperthwaite et al. 2016), and are pursuing analyses on GW170104 and GW170814. One of aims of DESGW is to establish standard sirens (Schutz 1986, Holz \& Hughes 2005) as a cosmological tool. The poster by Butler et al., and the talk by Herner et al., these proceedings, have more details on our BBH observations and DESGW.

The strength of the DECam GW-EM Collaboration is the wide array of telescopes that can be brought to bear on a GW event. For GW170817 we obtained data across the electromagentic spectrum: in radio the VLA and ALMA; in the optical and NIR the Hubble Space Telescope $2.4 \mathrm{~m}$, the Blanco $4 \mathrm{~m}$, the SOAR $4 \mathrm{~m}$, the Gemini $8 \mathrm{~m}$, the Magellan $8 \mathrm{~m}$; in the x-ray the Chandra spacecraft. Our collaboration has long experience following up short gamma ray burst events, and the expected optical counterparts of neutron star merger events (see, e.g., Metzger \& Berger 2012, Berger 2014). 
Annis

\section{Search and Discovery}

On August 17, 2017 the LIGO/Virgo GW detectors recorded the loudest event yet, a long, high frequency waveform. The interpretation was of a binary neutron star merger of $1.5+1.3 M_{\odot}$, resulting in a merger remnant of $2.7 M_{\odot}$, heard from a distance of $40 \mathrm{Mpc}$. As the three detector network was operating, the spatial localization area was small (Abbott et al. 2017f).

An optical and infrared counterpart to the NS event GW170817 was independently detected by several teams within the next $12 \mathrm{hr}$ (Abbott et al. 2017g), including ours (Allam et al. 2017), at a location deep inside the bright galaxy NGC 4993. In our collaboration, the source was found via a visual inspection of nearby galaxies in our raw data stream by Ryan Chornock. The discovery by DESGW initiated a follow-up program by our collaboration that lasted until the counterpart was lost behind the sun two weeks later. (Soares-Santos et al. 2017).

We performed a search for all possible counterparts in our data. Our search is designed to find kilonova as predicted in, e.g., Barnes \& Kasen 2013. We imaged $70 \operatorname{deg}^{2}$ in $i$ and $z$ bands, in two tilings of 18 hexes at 90 sec in each, deep enough to find kilonova out to $>100$ Mpc despite the uncertainties in the predicted luminosities of kilonova. The

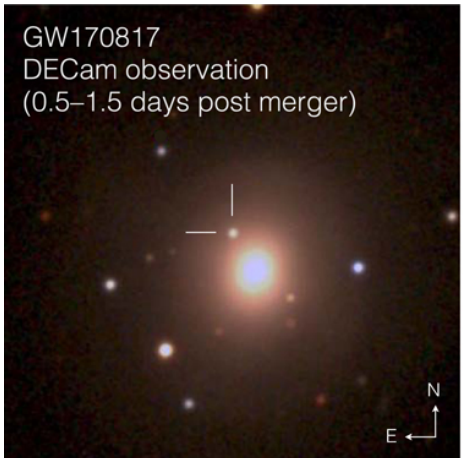

\section{GW170817}

DECam observation

(>14 days post merger)

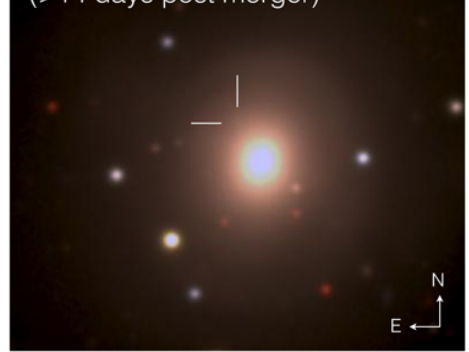

Figure 1. The DESGW discovery image of the NGC4993 kilonova (Soares-Santos et al. 2017).

search area was only $40 \%$ covered by pre-existing DECam images and we acquired the rest August 31 to September 2. We expect any kilonova-like transients to have faded below detection thresholds by that time, $>2$ weeks after the trigger. The camera fill factor is $80 \%$, leaving $4 \%$ area loss after two tilings. Weather on the nights of template observations was partially clouded and caused a 3\% loss. Processing failures accounted for less than $1 \%$ loss. The final area included in the search was 65 square degrees. Our difference imaging results in 1500 transient candidates with magnitudes between 15.5 and 20.5, where a candidate means on object detected on at least two search images. The magnitude cutoff was limited by the depth of the template images: $\mathrm{i}=21.2$ and $\mathrm{z}=$ 20.5, which can be compared with the depth of the search images: $i=22.0$ and $z=21.3$. This list of transients was cleaned by a set of cuts designed to reject moving objects, background artifacts and long-lived transients:

(a) The candidate must have at least one detection in $\mathrm{i}$ and one detection in $\mathrm{z}$ band.

(b) The candidate must pass our machine learning based junk rejection threshold. This rejects non-astrophysical artifacts such as dipoles. The efficiency of this criterion as measured from point sources injected into our images is $\sim 90-100 \%$ for both $\mathrm{i}$ and $\mathrm{z} \sim 17$ - 22 mag.

(c) The candidate must have faded significantly between the search (first) and template (last) observations. Specifically, we require the change in the candidate flux to be greater, at $3 \sigma$ level, than the flux in a circular aperture of radius $=5$ pixels on the template image. This criterion eliminates slow-evolving transients (e.g., supernovae). 
The initial list of 1500 candidates are cut down to 252 by the first cut, down to 81 candidates by the second cut, and down to one candidate by the third cut. This candidate is the optical transient in NGC4993. We therefore conclude that the counterpart to GW170817 is the new object discovered in NGC 4993. (Soares-Santos et al. 2017)

The first DECam images of the optical counterpart to the GW170817 at 11.40 hours postmerger showed an object with $i=17.30 \mathrm{mag}$ and $(i-z)=$ 0.13 , bright and nearly flat spectrum, with an absolute magnitude of $\mathrm{M}_{i}=15.7$. For the first few days the optical data are consistent with a blackbody, and for the first few days the data in each bandpass falls $m \propto t^{-n}$, where $n$ ranges from 2 in the $u$-band to 0.4 in the $Y$-band. The object fades much faster in the blue than it does in the red. Our observations

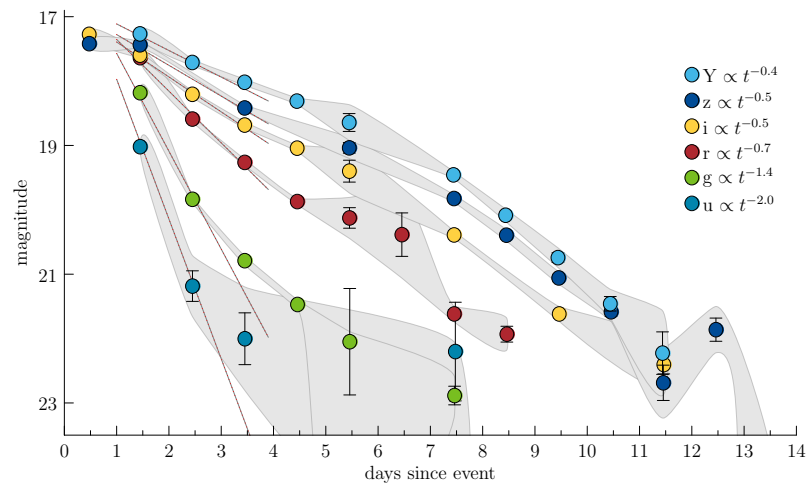

Figure 2. DECam observations of the counterpart in NGC 4993 for the two weeks after the merger (SoaresSantos et al. 2017).

on day 0.6 and on day 1.5 show that the peak of the light curve of this event was at $\approx 1$ day. At $\mathrm{M}_{i}=15.7$, the optical transient is bright enough for us to detect it out to 425 Mpc. (Soares-Santos et al. 2017)

\section{NGC 4993: The Host Galaxy}

We used DECam (Palmese et al. 2017) and Hubble Space Telescope data (Blanchard et al. 2017), along with archival data, to study the host galaxy of GW170817. The HST data in particular rule out a point source at the location of the counterpart brighter than $M_{V}=-7$ as recently as 4 months prior to the merger; the eliminates supergiants as a progenitor and likely globular clusters as the site of the merger as half the globular cluster population would be brighter than this.

The NGC 4993 is an elliptical galaxy that in $\mathrm{i}$-band is well described by a $n \approx 4$ Sersic profile. The offset of the counterpart from the nucleus of NGC 4993 is $2.1 \mathrm{kpc}$, inside the galaxy's halflight radius. From modeling of the UV to MIR SED we infer the star formation history, and find an exponentially declining SFH, with a median stellar popula-

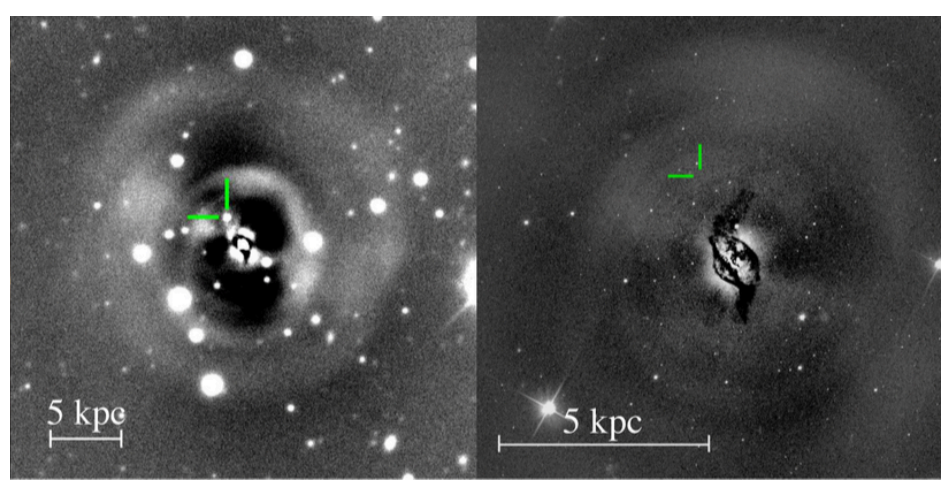

Figure 3. Shell, left, and central dust structure, right of NGC 4993. The counterpart location is marked (Palmese et al. 2017). tion age of $11.2_{-1.4}^{+0.7} \mathrm{Gyr}$. The present-day SFR is low, $\approx 0.01 \mathrm{M}_{\odot} \mathrm{yr}^{-1}$. Assuming that the star formation history is the probability distribution function for the binary birth age, the median merger timescale 
is $11.2_{-1.4}^{+0.7}$ Gyr with a $90 \%$ probability the BNS system formed between 6.813 .6 Gyr ago. Assuming a circular orbit and equal neutron star masses of $1.4 \mathrm{M}_{\odot}$, this corresponds to an median initial separation of $4.5 \mathrm{R}_{\odot}$. The resulting initial separation and eccentricity constraints are consistent with current galactic binary NS systems. Given the long merger timescale and small projected offset we conclude that the binary system experienced at most a modest natal kick, with an upper limit of $200 \mathrm{~km} / \mathrm{sec}$. (Blanchard et al. 2017).

The DECam data shows NGC 4993 has clear and significant shell structure. Shell structure in indicative of a minor merger, in which the smaller galaxy has $<10 \%$ of the mass of the larger galaxy. The HST data shows complex dust lanes in the galactic center. A spectrum from $6 \mathrm{dF}$ shows narrow $\mathrm{H}_{\alpha}, \mathrm{H}_{\beta}$, OIII, \& NII lines whose line ratios indicate the presence of a $\mathrm{AGN}$, as does the x-ray and radio luminosites of the center. These indicate that the smaller galaxy was dust and gas rich in comparison with NGC 4993, and the merger transported these to the center while the stars formed the shells. The position of the counterpart on or near one of the shells; there is no star formation occurring there. The merger likely happened 28 Myr ago. These features are uncommon in ellipticals, and it is interesting that the NS merger happened in such a galaxy. (Palmese et al. 2017).

\section{The Counterpart as a Short GRB Afterglow}

Two seconds after the GW merger event, two spacecraft carrying gamma ray detectors, Fermi's GBM and INTEGRAL's SPI-ACS, detected a short gamma ray burst (sGRB), and thus the collaborations operating them confirmed binary neutron star mergers as a progenitor of sGRBs. (Abbott et al. 2017h).

We obtained x-ray data on the counterpart using the Chandra spacecraft Margutti et al. 2017 and radio data from the Very Large Array and ALMA Alexander et al. 2017 to make observations of the sGRB afterglow.

The sGRB afterglow is broad brand synchrotron emission, and observations from radio to x-ray can constrain the spectral energy distribution and hence the physical properties of the event. We obtained the first x-ray detection from GW170817 using the Chandra spacecraft (Margutti et al. 2017), establishing a rising x-ray light curve. We also obtained extensive radio observations of GW170817 at centimeter and millimeter wavelengths using the VLA and ALMA, including the earliest observations taken in these bands. (Alexander et al. 2017). Combined as a function of time, three things stand out: 1) An on-axis afterglow emission similar to that typically observed in $\operatorname{sGRBs}\left(E_{k, i s o} \sim 10^{51} \mathrm{erg}\right)$ is clearly ruled out; 2) A late on-axis or isotropic afterglow onset due to the deceleration of a mildly relativistic outflow is consistent with the $\mathrm{X}$-ray observations but not the radio limits; 3) The radio and x-ray light curves are consistent with an off-axis relativistic jet with an energy of $10^{49}-10^{50} \mathrm{erg}$ and opening angle of $\theta_{j}=15^{\circ}$ expanding into a low-density medium of $\sim 10^{-4}-10^{-2} \mathrm{~cm}^{-3}$, which we are viewing at $\theta_{\text {obs }} \approx 20-40^{\circ}$. We argue that GW170817 is the first detection of an off-axis afterglow from a sGRB. (Margutti et al. 2017, Alexander et al. 2017).

The sGRB that followed GW170817 was subluminous: it occurred closer than any other sGRB with a known $z$, and had an energy $1,000 \times$ smaller than the next two closest sGRB with known $z$. (Abbott et al. 2017h). Our radio and x-ray observations allow us to place the afterglow in the context of other sGRB afterglows. In the x-ray, the counterpart to GW170817 is 3,000× less luminous than the median of the sGRB population, and $50 \times$ less luminous than the previous lowest. In the radio, the countpart's luminosity is $10000 \times$ less then the median of the population, and $500 \times$ less than the previous lowest. On the other hand, the physical properties of the jet and environment inferred from 
the joint radio and x-ray modeling are strikingly similar to those of the rest of of the sGRB population. The clear inference is that the primary difference between the sGRB associated with GW170817 and the rest of the population is the effect of viewing angle: we are viewing it off-axis. If the counterpart had been at the redshifts usual for sGRB, it would not have been detected with the current generation of radio and x-ray telescopes. (Fong et al. 2017)

We are thus able to compare the cosmological population of short GRBs to a local analog. NGC 4993 has higher stellar population age and stellar mass but lower star formation rate then the rest of the sGRB host population. The counterpart to GW170817 is closer to the center of NGC 4993 than $70 \%$ of the sGRB population to their hosts. The progenitor of GW170817 may have had a comparatively small kick velocity or perhaps just happened to be near pericenter of its orbit at the time of merger (Fong et al. 2017). NGC4993 stands out from the population of sGRB hosts as less asymmetric and more concentrated. sGRB hosts tend to be late type galaxies. Clear major galaxy mergers are unusual amongst sGRB hosts. However, it is entirely plausible that many of them have undergone a recent minor galaxy merger that has increased their asymmetry. (Palmese et al. 2017).

\section{Observations of the Kilonova}

In order to understand the nature of the counterpart we obtained optical spectra using the $4 \mathrm{~m}$ SOAR \& $6.5 \mathrm{~m}$ Magellan telescopes, and the $2.4 \mathrm{~m}$ HST STIS instrument (Nicholl et al. 2017), and infrared spectra from the 8m Gemini telescope (Chornock et al. 2017) of the counterpart to understand the nature of the light emission.

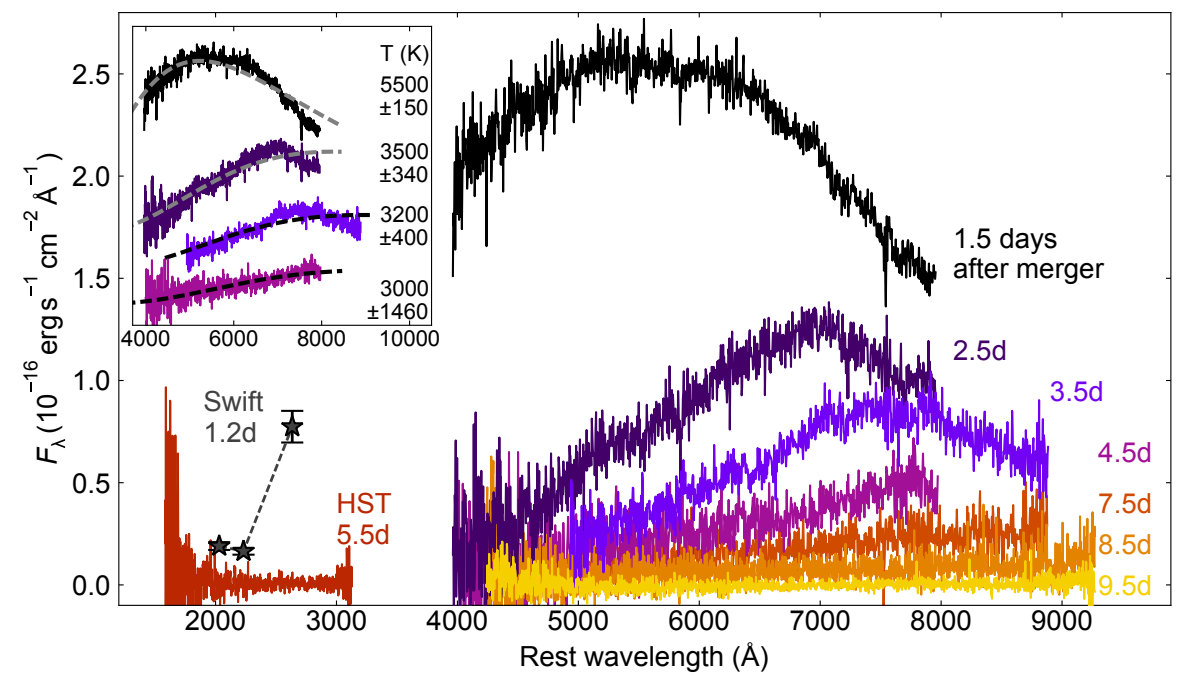

Figure 4. Optical spectroscopy of the counterpart for the first 10 days after merger. (Nicholl et al. 2017)

Figure 4 shows the high cadence optical spectra. Spectra at 7.5 days and earlier are from SOAR, those later are from Magellan. The source spectrum at 1.5 days after merger is a featureless near-blackbody of $\mathrm{T}=5000 \mathrm{~K}$. Over the next 24 hours the source cools by $1500 \mathrm{~K}$, and by day 4.5 the peak of the spectrum is in the infrared, $\lambda>800 \mathrm{~nm}$. The spectra remain featureless throughout the observation period. The spectra are too red to 


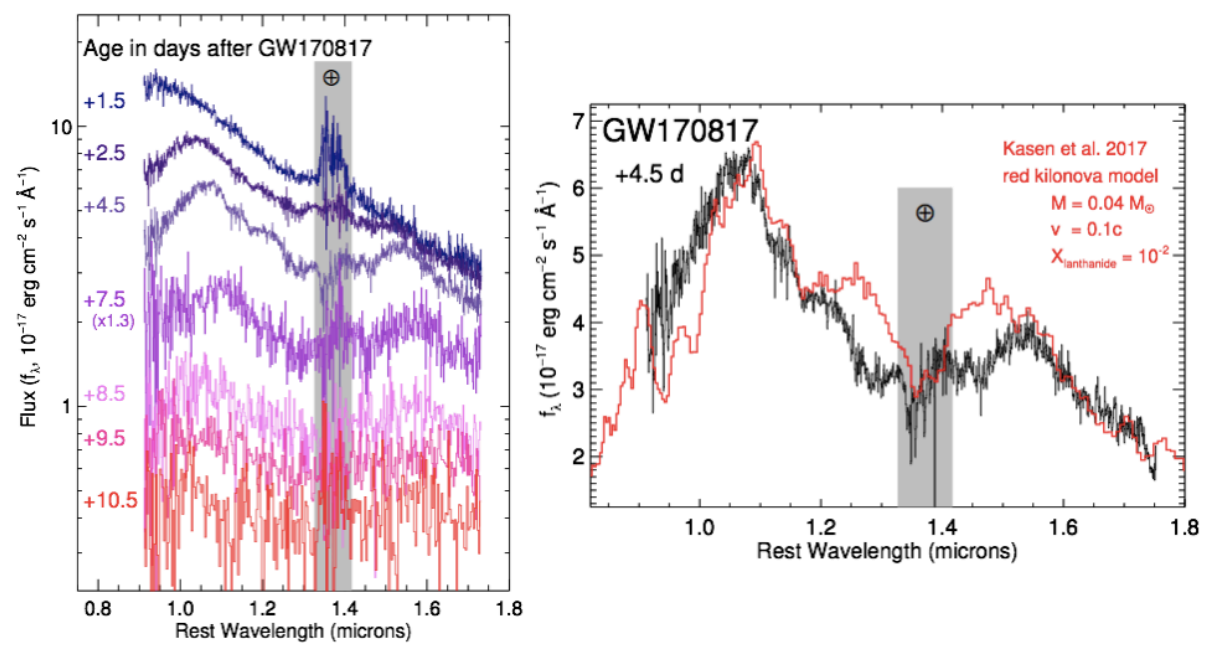

Figure 5. On the left: NIR specta of the counterpart for the first 10 days after merger. On the right, the day 4.5 Gemini NIR spectra with the best fit kilonova model overplotted. (Chornock et al. 2017)

be consistent the synchrotron emission generally seen in sGRB afterglows and too bright to be consistent with the modeling of the afterglow in Margutti et al. 2017. The spectra are too red to be consistent with supernova spectra, and even supernova with expansion velocities of $\approx 0.1 c$ have highly featured spectra. The spectra of the counterpart are notably featureless. The spectra can be modeled by kilonova model spectra (Kasen et al.(2013), Kasen et al. 2017) if the Lanthanide fraction is very low $\left(\lesssim 10^{-4}\right)$, to make the spectra sufficiently blue, and the expansion velocities are $\approx 0.3 \mathrm{c}$, to remove structure from the spectrum. (Nicholl et al. 2017).

Figure 5 shows the high cadence NIR spectra. The source spectrum at 1.5 days after merger is smooth and near-linear. Over the next 24 hours a peak appears at $1 \mu \mathrm{m}$ and by 4.5 days after merger another peak at $1.5 \mu \mathrm{m}$ is prominent. source cools by $1500 \mathrm{~K}$, and by day 4.5 the peak of the spectrum is in the infrared, $\lambda>800 \mathrm{~nm}$. This evolution from a smooth blue continuum to a redder continuum with two dominant peaks suggests a transition from one source opacity to another. The day 4.5 spectrum is particularly informative, as seen on right in Figure 5 . When we fit the day 4.5 spectrum with models from Kasen et al. 2017, we find the best fit model has a high Lanthanide fraction, $\approx 10^{-2}$ and an expansion velocity of $0.1 \mathrm{c}$. All of the spectral models of kilonova, from the early (Kasen et al.(2013), Hotokezaka et al.(2013)) to the latest (Kasen et al. 2017, Tanaka et al. 2017) models have the basic features of peaks near 1.1 and $1.55 \mu \mathrm{m}$, and agree that these indicate the presence of $r$-process nucleosynthesis elements as the dominant opacity source, as opposed to a spectral peak in the optical for material dominated by iron-peak opacities. The agreement between model and data is astonishingly good, given the uncertainties in the underlying atomic data and the simplifications inherent to a single-component model. (Chornock et al. 2017)

The $i-z$ color and $M_{i}$ from the first DECam data are consistent with a $\mathrm{T}=8300 \mathrm{~K}$ blackbody with a luminosity of $\lambda L_{\lambda} \approx 5 \times 10^{41} \mathrm{erg} / \mathrm{sec}$. At 0.6 days since merger, this implies an expansion velocity of $0.3 \mathrm{c}$. This is consistent with the optical spectra obtained in the following few days. The infrared spectra require a different component, expanding closer to $0.1 \mathrm{c}$. We fit spectral models of kilonova as a function of ejecta mass, ejecta velocity, and opacity/Lanthanide fraction to light curves of $u, g, r, i, z, Y$ DECam and 
$H, K_{s}$ Gemini-S FLAMNGOS-2 photometry over the two weeks subsequent to the merger event using the MOSFit light curve modeling program. Single component models did not describe the data, as expected. The best fitting two component and best fitting three component models were indistinguishable from an information criteria score; Occam's razor suggests we select the two component model. The best fitting model had

- blue component: $\mathrm{M}_{e j}=0.014_{-.001}^{+.002} \mathrm{M}_{\odot}, v / c=0.266_{-.002}^{+.007}, \& \kappa \equiv 0.5 \mathrm{~cm}^{2} / \mathrm{gm}$

- red component: $\mathrm{M}_{e j}=0.036_{-.002}^{+.001} \mathrm{M}_{\odot}, v / c=0.123_{-.014}^{+.012}, \& \kappa=3.35_{-.34}^{+.36} \mathrm{~cm}^{2} / \mathrm{gm}$

Matching the derived Galactic r-process production rate requires kilonova ejecta masses in the range of $0.01 \quad 0.1 \mathrm{M}_{\odot}$ given estimates of the binary NS merger rate from the Galactic neutron star population. Our best fit two-component model has a total ejecta mass of $0.055 \mathrm{M}_{\odot}$, with $0.035 \mathrm{M}_{\odot}$ of material enriched in the heavy r-process, squarely in the middle of the range. These data are clear evidence that binary NS mergers are the site of r-process nucleosynthesis. (Cowperthwaite et al. 2017)

The data presented in this set of papers represent by far the best observations of an r-process powered kilonova, and it is remarkable how well the observations match theoretical models.

\section{Measuring the Hubble Constant with GW}

The GW amplitude from a source is predominately set by $\frac{M}{d} \cos \theta_{o b s}$. The chirp mass $M$ can be constrained by the GW frequency and its derivatives, leaving as the main unknowns the distance and inclination angle, $\theta_{o b s}$. This standard siren proposal (Schutz(1986) \& Holz \& Hughes(2005)) is most powerful when the $z$ of a counterpart to the GW event can be measured.

The redshift of NGC 4993 is known; our search of the LIGO/Virgo spatial localization area did not depend on the GW distance or host $z$; therefore the redshift of the source can be used as an independent variable in the joint cosmological analysis. The $z$ of NGC 4993 is small enough that the Hubble expansion does not dominate. NGC 4993 is part of a small group of galaxies; the average $z$ of the group is a better estimator of the Hubble expansion. The group itself is streaming towards the Great Attractor and a correction for this peculiar velocity is made based on peculiar velocity maps over large parts

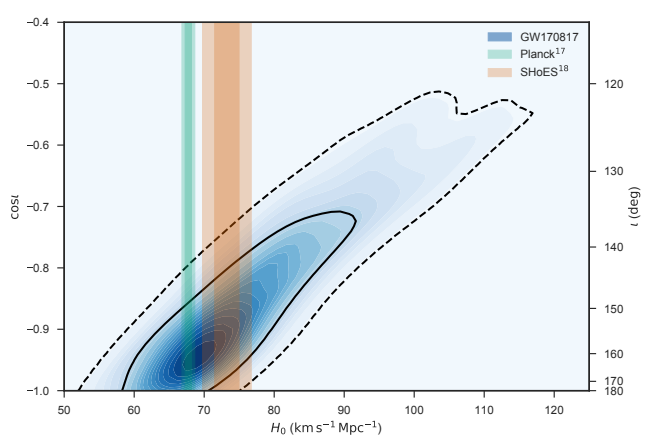

Figure 6. The posterior density for $\mathrm{H}_{0}$ and $\cos \theta_{\text {obs }}$ is shown in blue contours. The brown band is the astrophysical distance ladder constraint on $\mathrm{H}_{0}$, while the green band is the cosmological inverse distance ladder constraint. (Abbott et al. 2017b) Since inception our collaboration has included the Holz LIGO Group at Chicago: Dan Holz, Hsin-Yu Chen, Ben Farr, Zoheyr Doctor. of the sky. The resulting Hubble expansion is $z=0.0101 \pm 0.0005$. The Hubble Constant is, for small $z, H_{0}=c z / d$. The GW signal from GW170817 was the highest yet, at $\mathrm{S} / \mathrm{N}=32$ and the distance constraints are relatively strong. Further, the GW polarization information from having a third GW detector, Virgo, constrains $\theta_{\text {obs }}$ to $144^{\circ}-180^{\circ}$. The distance measured is $d=43.8_{-6.9}^{+2.9}$ Mpc. A Bayesian analysis inferred a posterior distribution on $\mathrm{H}_{0}$ and $\theta_{o b s}$, marginalized 
over uncertainties in $z$, and found $\mathrm{H}_{0}=70_{-8}^{+12} \mathrm{~km} / \mathrm{s} / \mathrm{Mpc}$. Figure 6 shows the result of this analysis, illustrating the GW degeneracy between distance and $\theta_{o b s}$. This is the first step to the era of percent-level gravitational-wave cosmology. (Abbott et al. 2017b)

If one could place constraints on $\theta_{\text {obs }}$ from EM observations, the uncertainty on the GW distance can be lessened. We made an investigation of this using by modeling the off-axis jet using the x-ray and radio emission up to 40 days after the merger. Because of the shape of the $\mathrm{H}_{0}-\cos \theta_{\text {obs }}$ degeneracy, the result of our analysis, $\theta_{\text {obs }} \approx 25^{\circ}-50^{\circ}$, doesn't significantly lessen the uncertainties on the $\mathrm{H}_{0}$ measurement, but it does shift the maximum a posterior value of $\mathrm{H}_{0}$ to higher values. (Guidorzi et al. 2017).

\section{Summary}

The DECam on the Blanco $4 \mathrm{~m}$ telescope was used to both perform an independent discovery of the counterpart to GW170817 and to eliminate other possible candidates in the spatial localization area. Our collaboration studied the host galaxy, the radio and x-ray afterglow, and the lightcurve and optical and NIR spectroscopy of the counterpart. Modeling of the DECam light curve provides a concrete two-component model for the kilonova with $0.01 \mathrm{M}_{-} \odot$ ejected with low opacity and high velocity, and $0.04 \mathrm{M}_{\odot}$ ejected with high opacity and low velocity. With the identity of the counterpart secure, the first measurement of $\mathrm{H}_{0}$ using gravitational wave standard sirens was performed.

\section{References}

Abbott, B. P., Abbott, R., Abbott, T. D., et al. Nature, 551:85-88, 2017

Abbott, B. P., Abbott, R., Abbott, T. D., et al. Physical Review Letters, 119(16):161101, 2017

Abbott, B. P., Abbott, R., Abbott, T. D., et al. Astrophysical Journal Letters, 848:L12, 2017

Abbott, B. P., Abbott, R., Abbott, T. D., et al. Astrophysical Journal Letters, 848:L13, 2017

Alexander, K. D. , Berger, E., Fong, et al. Astrophysical Journal Letters, 848:L21, 2017

Allam, S., Annis, J., Berger, E., et al. GCN 21530, 2017

Annis, J., Soares-Santos, M., Berger, E., et al. Astrophysical Journal Letters, 823:L34, 2017

Barnes, J., \& Kasen, D. Astrophysical Journal, 775:18, 2013.

Berger, E., Annual Reviews of Astronomy and Astrophysics, 52, 43, 2014.

Blanchard, , P. K. , Berger, E., Fong, et al. Astrophysical Journal Letters, 848:L22, 2017

Chornock, R., Berger, E., Kasen, D., et al. Astrophysical Journal Letters, 848:L19, 2017

Cowperthwaite, P. S., et al. Astrophysical Journal Letters, 826:L29, 2016

Flaugher, B., Diehl, H. T., et al., and DES Collaboration. Astronomcial Journal, 150:150, 2015.

Fong, W., Berger, E., Blanchard, P. K., et al. Astrophysical Journal Letters, 848:L23, 2017

Guidorzi, C., Margutti, R., Brout, D, et al. ArXiv e-prints, October 2017.

Holz, D. E., \& Hughes, S. A. Astrophysical Journal, 629:15, 2005.

Hotokezaka, K., Kiuchi, K., Kyutoku, K., et al. Physical Review D, 87:024001, 2013.

Kasen, D., Metzger, B., Barnes, J., et al. Nature, 551:80-84, November 2017.

Kasen, D., Badnell, N. R., \& Barnes, J. Astrophysical Journal, 774:25, 2013.

Margutti, R., Berger, E., Fong, W., et al. Astrophysical Journal Letters, 848:L20, 2017

Metzger, B. D., \& Berger, E. Astrophysical Journal, 746:48, 2016.

Nicholl, M., Berger, E., Kasen, D., et al. Astrophysical Journal Letters, 848:L18, 2017

Palmese, A., Hartley, W., Tarsitano, et al. Astrophysical Journal Letters, 849:L34, 2017

Schutz, B. F. Nature, 323:310, 1986.

Soares-Santos, M., Kessler, R., Berger, E., et al. Astrophysical Journal Letters, 823:L33, 2016

Soares-Santos, M., Holz, D. E., Annis, J., et al. Astrophysical Journal Letters, 848:L16, 2017

Tanaka, M., Utsumi, Y., Mazzali, P. A., et al. ArXiv e-prints, October 2017. 